\title{
The Spectrum of Motor Neuron Diseases: From Childhood Spinal Muscular Atrophy to Adult Amyotrophic Lateral Sclerosis
}

\author{
Stacey A. Sakowski ${ }^{1}$ - Eva L. Feldman ${ }^{1,2}$
}

Published online: 21 March 2015

(C) The American Society for Experimental NeuroTherapeutics, Inc. 2015

Key Words Amyotrophic lateral sclerosis · Motor neuron · Motor neuron disease $\cdot$ Spinal muscular atrophy

Since the topic of motor neuron diseases was last covered in Neurotherapeutics in 2008, increasing insight into genetic causes and underlying disease mechanisms has fueled several advances that have paved the way for new therapeutic strategies that are currently in development or being tested in clinical trials. In this special issue, we present reviews by leading investigators in the field that detail these advances for spinal muscular atrophy (SMA), an inherited neuromuscular disease associated with motor neuron degeneration and muscle atrophy that presents early in life, and amyotrophic lateral sclerosis (ALS), a fatal adult-onset neuromuscular disease also characterized by loss of motor neurons. The papers are divided into 4 main sections that cover 1) SMA advances, 2) ALS challenges and recent biological advances, 3) ALS clinical trial design considerations and outcome measures, and 4) emerging ALS treatments. Together, these articles offer a timely and comprehensive look into the recent research advances, current clinical considerations, and therapeutic prospects currently in development or being tested for motor neuron diseases.

The issue begins with 2 papers focused on SMA. In the first article, Drs. Michelle Farrar and Matthew Kiernan [1] provide a detailed overview of the genetic basis of SMA forms and variants and present common pathophysiological mechanisms, including discussion of the function of the survival

Eva L. Feldman

efedman@umich.edu

1 A. Alfred Taubman Medical Research Institute, University of Michigan, Ann Arbor, MI 48109, USA

2 Department of Neurology, University of Michigan, 109 Zina Pitcher Place, 5017 AAT-BSRB, Ann Arbor, MI 48109, USA motor neuron protein. They then discuss considerations for therapeutic development and testing before reviewing potential molecular biomarkers for outcome assessments and trial design. Next, Drs. Constantin d'Y dewalle and Charlotte Sumner [2] present an eloquent synopsis of promising SMA treatments currently in advanced stages of development, a discussion that is prefaced by an overview of the available in vivo mouse SMA models. The treatment strategies include some originally designed for applications in ALS, approaches to induce survival motor neuron, and new treatment paradigms harnessing epigenetic targets, antisense oligonucleotides, and gene therapy approaches. Notably, both articles also discuss important issues that remain to be addressed for current progress, such as how well preclinical models recapitulate the human phenotype, how to factor in disease heterogeneity and identify and define potential therapeutic windows, and how to best analyze effects on disease progression. We believe the recent novel insights and current advances bode well for continued progress and the identification of much needed treatment options in SMA.

The second section of the issue begins with a review by Drs. Jeffrey Rosenfeld and Michael Strong [3], which specifies the current challenges underlying therapeutic development that accompany the recent expansions in our understanding of the complex, multifaceted nature of ALS. Briefly, establishing firm diagnostic and prognostic criteria, determining what role frontotemporal dysfunction plays in the classification of ALS, identifying an accepted biomarker, determining how multidisciplinary care affects phenotypic variability, and understanding how the genetic basis of ALS and gene-environment interactions contribute to pathogenesis are all key issues that need to be addressed. Along these lines, this section continues with articles highlighting the most recent genetic and biological advances in ALS. In the article by CooperKnock et al. [4], we hear about the advances gained from the discovery of the recent identification of a hexanucleotide repeat expansion in $\mathrm{C} 9$ orf 72 , and the authors emphasize the associated phenotypes, proposed disease mechanisms related 
to the repeat expansion, similarities to other disorders with repeat expansions, premise for genetic screening, and most promising therapeutic developments. Dr. Sami Barmada [5] then discusses data supporting a converging mechanism in ALS centered on RNA dysregulation, presenting the current evidence depicting abnormalities in RNA processing and metabolism, mislocalization or aggregation of RNA binding proteins, and mutations in RNA processing enzymes. He also identifies potential therapeutic targets based on these mechanisms that may foster novel treatment strategies. Next, the pathways associating TAR DNA-binding protein 43 mutations and pathological inclusions observed in ALS with disease pathogenesis are reviewed by Drs. Emma Scotter, HanJou Chen, and Christopher Shaw [6], and the potential of TAR DNA-binding protein 43-positive protein inclusions as therapeutic targets is also discussed. Finally, Drs. Kristopher Gray Hooten, David Beers, Weihua Zhou, and Stanley Appel [7] introduce the noncell-autonomous nature of ALS, specifically detailing the dual role of the immune system in disease onset and progression. They also discuss the current therapeutic strategies in development to enhance neuroprotection and combat toxic neuroinflammation. Collectively, the papers in this section present a detailed view of some of the most recent discoveries in the last decade regarding ALS pathogenesis, and also portray the implications of these advances and current resulting progress towards therapeutic development.

While enhancing our understanding of disease pathogenesis is of paramount importance to therapeutic development, also equally critical, as mentioned above, is the need to overcome the challenges that accompany clinical translation and testing of newly discovered therapeutic options in patients with ALS. The third section of this issue therefore includes a collection of articles detailing these challenges and trial design considerations that are important for continued progress. First, Drs. Katharine Nicholson, Merit Cudkowicz, and James Berry [8] present an overview of how improvements in clinical trial design are affecting the current therapeutic testing landscape. They emphasize the need to consider treatment delivery paradigms, subgroups among the heterogeneous ALS patient population, and potential trial outcome measures in ALS clinical research. They also review how the development of diagnostic, prognostic, and pharmacodynamic biomarkers, and the increasing application of adaptive trial designs are setting the stage for exciting progress in the clinical ALS research arena. The question of potential outcome measures is then outlined by Dr. Seward Rutkove [9]. He offers insight into the currently used approaches to assess disease phenotypes and ways to account for inherent intersubject variability, and further discusses recommendations and considerations for assessing clinical outcomes in future clinical trials. Likewise, Dr. Zachary Simmons [10] addresses the value of determining patient-perceived quality of life in addition to traditional physical outcome measures in ALS clinical trials. Specifically, he introduces the various available tools to assess subject wellbeing, and offers recommendations as to which tools are best fit for certain clinical testing paradigms to capture the true effect of the interventions being tested. Novel advances in neuroimaging are then reviewed by Drs. Esther Verstraete and Bradley Foerster [11], who present the clinical value of the various advanced neuroimaging modalities utilized to study ALS, discuss the potential of imaging to offer diagnostic and prognostic biomarkers, and introduce the remaining challenges underlying the translation and utility of neuroimaging in clinical applications and therapeutic trials. To end this section, Dr. Neta Zach et al. [12] highlight the tremendous benefits of the Pooled Resource Open-access ALS Clinical Trials (PRO-ACT) database, a platform that currently contains data on the thousands of patients with ALS participating in 17 completed clinical trials, and incorporation of new patient and trial data is ongoing. Briefly, this resource has enabled important research regarding clinical trial recruitment, enrollment, and outcome assessment considerations, and also promoted an opportunity to gain insight into ALS pathogenesis across the heterogeneous patient population. Together, the articles in this section lend support to the continued clinical treatment advances that are possible with improved trial designs and an enhanced understanding of patient classifications and clinical outcomes.

Finally, in the last section we present 2 papers that review emerging treatment strategies that are rapidly making their way through the clinical translation pipeline. We begin with an article by Drs. Linga Reddy and Timothy Miller [13] that details the recent advances in RNA-based therapeutics for ALS and emphasizes the current progress in the preclinical and early clinical applications of antisense oligonucleotides and small interfering RNAs. The final paper, by our research group, then reviews the current progress in the development and application of stem cell-based therapies in ALS [14]. Briefly, we present preclinical data examining the therapeutic utility of various stem cell types and an overview of the paradigms that have progressed to early clinical trials; we include a brief update on the recent progress of our own clinical trial examining intraspinal transplantation of neural progenitor cells in patients with ALS. Overall, while most of the studies are small and not designed to determine efficacy, the results of the preclinical and early clinical studies support the continued development of stem cell therapies for ALS. We believe that with continued attention to issues such as selecting correct patient subpopulations, overcoming diagnostic delays, determining informative outcome measures, and improving trial design, we are poised for continued progress in both RNAbased and cellular therapies in ALS.

In summary, the emerging mechanistic themes amongst SMA and ALS, including alterations in RNA metabolism, loss of connectivity along the neuromuscular axis, and defects in axonal transport, are facilitating an enhanced understanding 
of the pathogenesis underlying these diseases affecting motor neurons, as well as the identification of novel therapeutic targets and treatment strategies. Likewise, continuing improvements in our ability to diagnose, characterize, and assess patient well-being are enhancing our ability to design informative, powerful clinical trials. Although questions still remain regarding potential therapeutic windows and how to address the multifaceted treatment demands of the complex and heterogeneous nature of motor neuron diseases, recent years have brought a wave of advances in our ability to design wellplanned and informative clinical trials that are fueling an incredible pace of therapeutic discovery for SMA and ALS. The current articles provide an excellent evaluation of these advances, and we greatly appreciate the time and effort of both the authors and reviewers, as their collective efforts and contributions have resulted in a spectacular, informative issue.

We would also like to thank Dr. Maral Mouradian, the Editor-in-Chief of Neurotherapeutics, Linda Powell, the Editorial Manager of Neurotherapeutics, and Saraswathi Sabapathy, from the Springer Journal Editorial Office, for the invitation to oversee this exciting issue and for their assistance and support.

\section{References}

1. Farrar M, Kiernan M. The genetics of spinal muscular atrophy: progress and challenges. Neurother 2015. doi:10.1007/s13311014-0314-x (in this issue).

2. d'Ydewalle C, Sumner C. Spinal muscular atrophy therapeutics: Where do we stand? Neurother 2015. doi:10.1007/s13311-0150337-y (in this issue).
3. Rosenfeld J, Strong M. Challenges in the understanding and treatment of amyotrophic lateral sclerosis/motor neuron disease. Neurother 2015. doi:10.1007/s13311-014-0332-8 (in this issue).

4. Cooper-Knock J, Kirby J, Highley R, Shaw PJ. The spectrum of C9orf72-mediated neurodegeneration and amyotrophic lateral sclerosis. Neurother 2015. doi:10.1007/s13311-015-0342-1 (in this issue).

5. Barmada S. Linking RNA dysfunction and neurodegeneration in amyotrophic lateral sclerosis. Neurother 2015. doi:10.1007/ s13311-015-0340-3 (in this issue).

6. Scotter E, Chen H-J, Shaw C. TDP-43 proteinopathy and ALS: Insights into disease mechanism and therapeutic targets. Neurother 2015. doi:10.1007/s13311-015-0338-x (in this issue).

7. Hooten KG, Beers D, Zhou W, Appel S. Protective and toxic neuroinflammation in amyotrophic lateral sclerosis. Neurother 2015. doi:10.1007/s13311-014-0329-3 (in this issue).

8. Nicholson K, Cudkowicz M, Berry J. Clinical trial designs in amyotrophic lateral sclerosis: Does one design fit all? Neurother 2015. doi:10.1007/s13311-015-0341-2 (in this issue).

9. Rutkove S. Clinical measures of disease progression in amyotrophic lateral sclerosis. Neurother 2015. doi:10.1007/s13311-0140331-9 (in this issue).

10. Simmons Z. Patient-perceived outcomes and quality of life in ALS. Neurother 2015. doi:10.1007/s13311-014-0322-x (in this issue).

11. Verstraete E, Foerster B. Neuroimaging as a new diagnostic modality in amyotrophic lateral sclerosis. Neurother 2015. doi:10.1007/ s13311-015-0347-9 (in this issue).

12. Zach N, Ennist DL, Taylor AA, et al. Being PRO-ACTive: What can a clinical trial database reveal about ALS? Neurother 2015. doi: $10.1007 / \mathrm{s} 13311-015-0336-\mathrm{z}$ (in this issue).

13. Reddy L, Miller T. RNA-targeted therapeutics for amyotrophic lateral sclerosis. Neurother 2015. doi:10.1007/s13311-015-0344-z (in this issue).

14. Goutman SA, Chen KS, Feldman EL. Recent advances and the future of stem cell therapies in amyotrophic lateral sclerosis. Neurother 2015. doi:10.1007/s13311-015-0339-9 (in this issue). 